\title{
PROFILE OF TRAUMA VICTIMS OF MOTORCYCLE ACCIDENTS TREATED AT HOSPITAL SÃO PAULO
}

\author{
Natália Miki ${ }^{1}$, ana Luiza Cabrera Martimbianco ${ }^{1}$, Lúcia Tomomi Hira ${ }^{1}$, Gisele Landim Lahoz ${ }^{1}$, Hélio Jorge Alvachian Fernandes ${ }^{1}$, \\ FERNANDO BALDY DOS REIS ${ }^{1}$
}

\begin{abstract}
Objective: To evaluate retrospectively, through analysis of medical records, the epidemiological aspects of patients traumatized by motorcycle accidents treated at the orthopedics ward, Hospital São Paulo, Brazil. Methods: It is a retrospective observational study. The analysis of patients' medical records comprised the period from January 2008 to December 2009. The data checked were: age, gender, type of collision, type and location of fracture, treatment performed (conservative or surgical), type of surgery, cost of synthesis material and hospitalization, period of hospitalization and postoperative complications. After data collection, statistical analysis was performed. Results: We analyzed 381 victims involved in motorcycle accidents.
\end{abstract}

Patients were predominantly male (85\%), with a mean age of 30.7 years old. Referring to distribution and site of lesions, $75.5 \%$ of individuals had lower limb fractures and in $95.4 \%$ of the cases, the treatment of choice was surgery. Twenty-nine patients were rehospitalized due to postoperative complications, such as exposure and failure of synthesis material, wound infection, necrosis, osteomyelitis, and pseudoarthrosis. Conclusion: It was possible to identify useful characteristics for planning preventative strategies to reduce the rate of motorcycle accidents and redirect public investment in health. Level of Evidence III, Retrospective Study.

Keywords: Accidents, traffic. Epidemiologic studies. Public health.

Citation: Miki N, Martimbianco ALC, Hira LT, Lahoz GL, Fernandes HJA, Reis FB. Profile of trauma victims of motorcycle accidents treated at hospital São Paulo. Acta Ortop Bras [online]. 2014;22(4):219-22. Available from URL: http://www.scielo.br/aob.

\section{INTRODUCTION}

Injuries caused by motorcycle accidents make up a significant proportion of serious traffic accidents, being a significant cause of increased morbidity and mortality, especially among young males. Every day, nearly 3,000 people die and 30,000 are seriously injured in traffic accidents worldwide. Costs are high when considering developing countries where the vulnerable traffic users are pedestrians, cyclists and motorcyclists. In 2020, road traffic collisions tend to reach the third leading cause of death and disability in the world. ${ }^{1,2}$

In Brazil, in 1998, 15,232 motorcyclists, victims of traffic accidents were admitted in hospitals, and in 2004 this number has evolved to 27,388 , corresponding, therefore, to an increase of $79.8 \%$. $^{3-6}$ In the decade 1998/2008, the Mortality Information System of the Ministry of Health (SIM-MS) recorded a total of 38,23 deaths in the various types of car accidents, and tragically, motorcyclists point out, whose mortality increased $754 \%$ in the mentioned decade. ${ }^{7}$

In 1994, a study in New Zealand estimated the average hospital cost per patient of US\$5,170 (approximately R $\$ 8,690$ ) per person involved in a motorcycle accident. The same study showed that the lower limbs were the most commonly injured (43\%). ${ }^{8}$ By studying the injuries pattern in victims of motor vehicles road traffic accidents in general, and particularly of motorcycle accidents, it cannot be restricted to data coming from the frame of their mortality. However, studies which describe the different types, locations and degrees of severity of injuries in victims of traffic accidents, which were killed or not, besides few, are difficult to perform. 5,6,9

Trauma epidemiology is essential to describe the morbidity, disability and limitations, as well as defining the most important target for prevention regarding the severity of the injury. The prevention of accidents is much more efficient than treating injuries, and also much cheaper for society. Injuries caused by accidents are the society's responsibility, especially due to the frequent involvement of young and productive people. ${ }^{10-12}$ The present study aimed to analyze the victims profile of motorcycle accidents who were admitted to a tertiary referral center for trauma, by raising features such as trauma etiology, gender, age, injury pattern, types of treatment carried out, among other factors, in order to delineate the epidemiological profile of these individuals.

All the authors declare that there is no potential conflict of interest referring to this article.

1. Department of Orthopedics and Traumatology, Universidade Federal de São Paulo - Escola Paulista de Medicina (DOT - UNIFESP/EPM), São Paulo, SP, Brazil.

Work developed at Trauma Group, Department of Orthopedics and Traumatology, Universidade Federal de São Paulo - Escola Paulista de Medicina (DOT - UNIFESP/EPM), São Paulo, SP, Brazil.

Correspondence: Rua Borges Lagoa, 783, $5^{\circ}$ andar, Vila Clementino, São Paulo, SP, Brazil. n.miki@unifesp.br 


\section{METHODS}

This is a retrospective cross-sectional descriptive study that was conducted after the approval of the University of São Paulo Ethics Research Committee, at the Orthopedics and Traumatology Ward, Hospital São Paulo, Universidade Federal de São Paulo / Escola Paulista de Medicina, São Paulo, Brazil, from January 2008 to December 2009. The ward consisted of 26 beds of which 16 are intended to male patients and 10 to female, but not all of them were used by trauma victims.

There were selected for the study adults over the age of 18 , victims of trauma caused by motorcycle accidents, being excluded those with musculoskeletal disease prior to the accident. Data survey, conducted through analysis of medical records of patients checked for: age, gender, location and type of trauma, the injury mechanism, applied treatment (conservative or surgical), cost of synthesis materials and hospitalization, duration of hospitalization and postoperative complications.

\section{RESULTS}

Data was collected between January 2008 and December 2009, resulting a total of 381 adults (aged 18 or older), victims of accidents involving motorcycles. There was a predominance of males, with 324 individuals (85\%), with a mean age of 30.7 years old (range 18-75) and 57 females (15\%), with a mean age of 30.3 years old (range 18-74), and most of women were in the passenger position of the motorcycle. (Table 1)

Regarding the mechanisms of injury, 173 cases (45.4\%) resulted from collision (car and motorcycle), 165 (43.3\%) from falling of motorcycle, 43 (11.2\%) due to run over by motorcycle. (Figure 1) Regarding the distribution and site of injuries, it was observed that 210 out of $381(67.7 \%)$ individuals had more than two associated injuries, resulting in 594 injuries, of which 449 (75.5\%) were fractures in lower limbs: 173 (29.1\%) were fractures of the tibia (107 compound and 66 closed fractures); $52(8.75 \%)$ fibular fractures, $83(13.9 \%)$ femoral fractures (23 compound and 60 closed fractures), 60 (10.1\%) ankle fractures, 35 (5.9\%) bone foot fractures, 27 (4.5\%) hip fractures (acetabulum and pelvis) and 19 (3.2\%) fractures of the patella. Regarding upper limbs fractures there was a total of 145 $(24.5 \%)$ fractures occurred: $47(32.4 \%)$ radio fractures, 28 (19.3\%) humerus fractures, 39 (26.9\%) fractures of the ulna: $24(16.5 \%)$ fractures of the bones of the hand, $6(4.1 \%)$ fractures of the clavicle and one $(0.7 \%)$ fracture of the scapula (Figures 2 and 3)

Surgery has been the treatment of choice in most cases, having been performed 567 (95.4\%) surgical procedures: 377 (66.5\%) internal fasteners, 165 (29.1\%) external fixations and 25 (4 4\%) with other features (skeletal tractions, amputations, arthroplasties and tenorraphies). The remaining $4.5 \%$ represent 27 cases treated by immobilization with plaster. (Figura 4).

Table 1. Characteristics of individuals participating in the study.

\begin{tabular}{c|c|c}
\hline & Number of Patients & Mean Age (years old) \\
\hline Feminine & $57(15 \%)$ & 30.3 \\
\hline Masculine & $324(85 \%)$ & 30.7 \\
\hline
\end{tabular}

Twenty-nine individuals (7.61\% of total patients) who developed postoperative complications were readmitted: 2 cases by exposure of synthesis material, 7 for failure of synthesis material, 12 by wound infection, one due to necrosis, 5 by osteomyelitis and two due to pseudoarthrosis. (Figure 5)

The total cost of all procedures performed in those individuals together to hospitalization summed $\mathrm{R} \$ 1,101,028.71$.

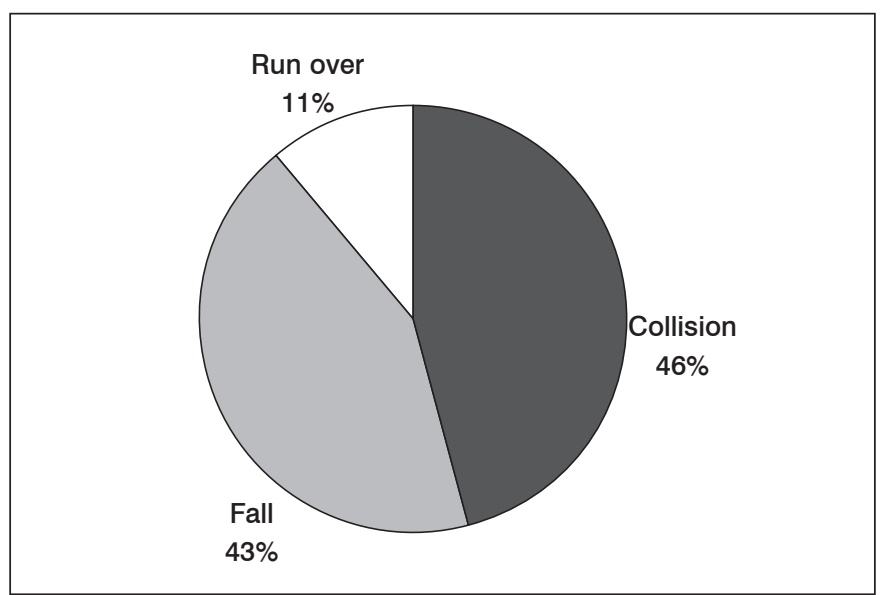

Figure 1. Injury mechanisms

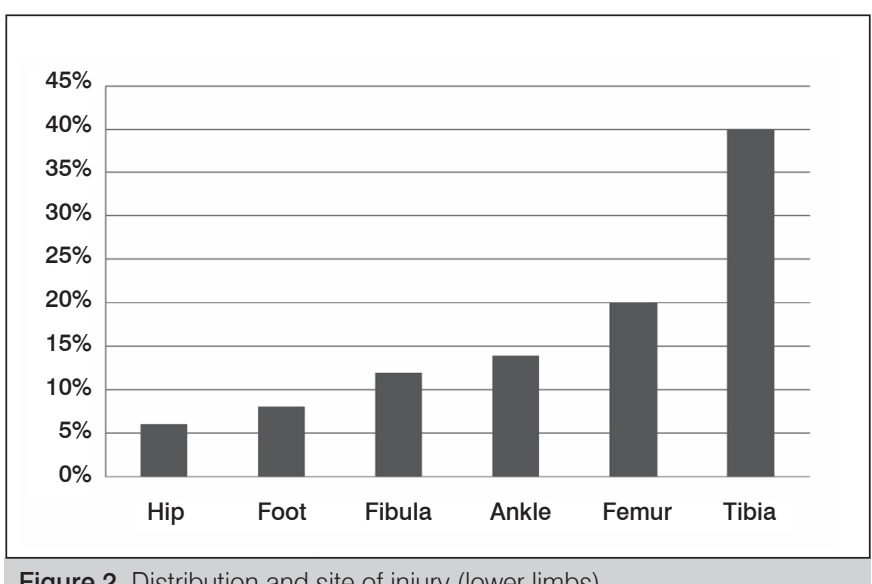

Figure 2. Distribution and site of injury (lower limbs).

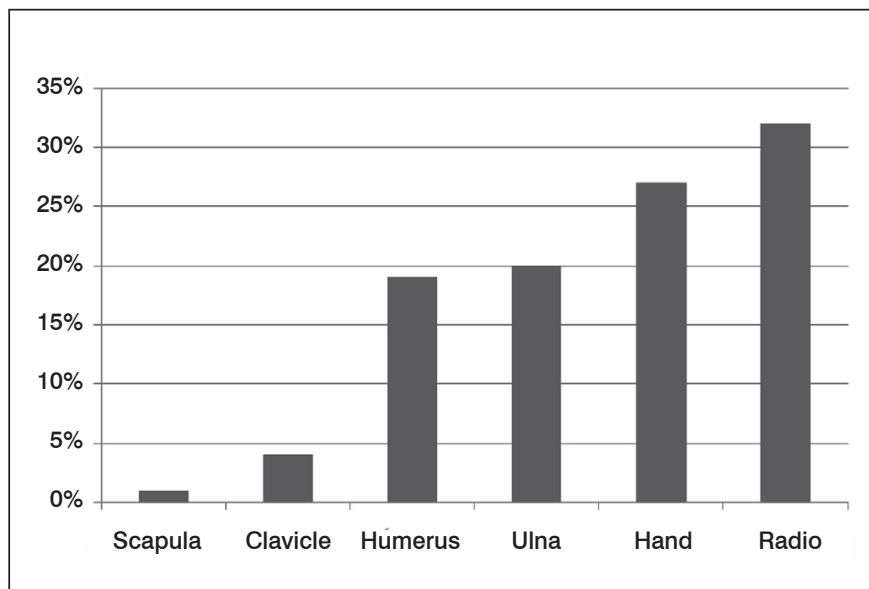

Figure 3. Distribution and site of injury (upper limbs). 


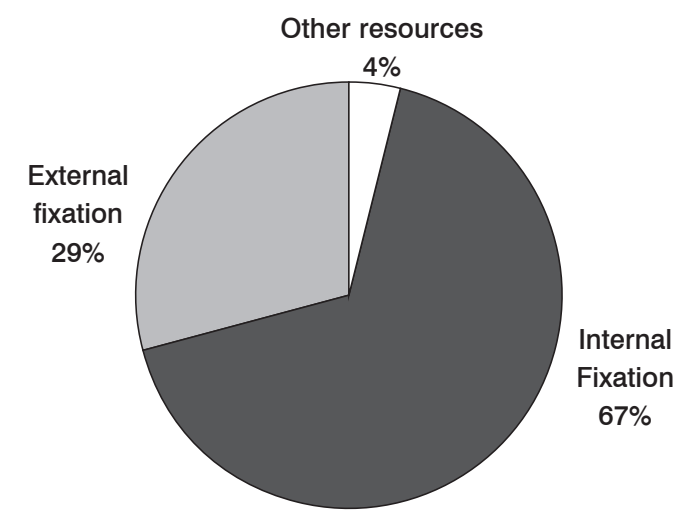

Figure 4. Treatment of choice.

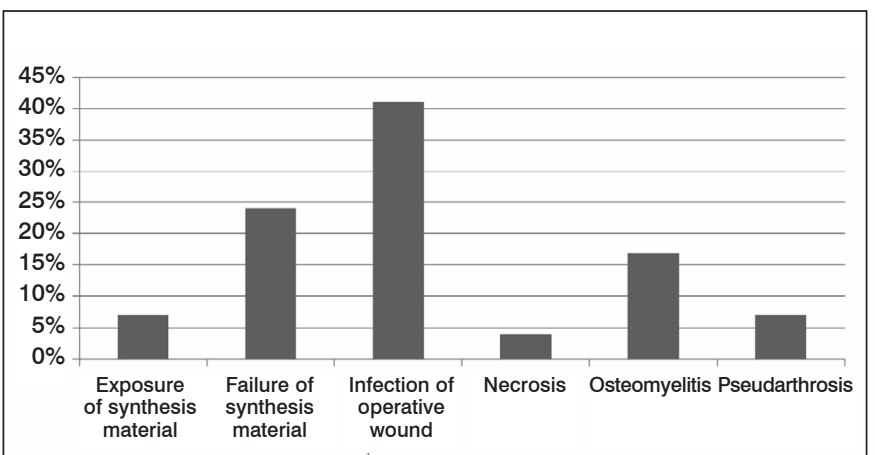

Figure 5. Postoperative complications.

\section{DISCUSSION}

In recent decades, the city of São Paulo, Brazil, has been showing alarming rates of traffic accidents, becoming nowadays a public health issue. Among these accidents are those involving motorcycles, whose fleet increases daily due to its low cost and agility, being mainly widespread use as a working tool. The purpose of this study was to analyze the epidemiological profile of individual victims of motorcycle accidents treated in the Orthopaedics and Traumatology Ward, Hospital São Paulo (Universidade Federal de São Paulo / Escola Paulista de Medicina, São Paulo, Brazil).

The increased use of motorcycle as a working tool can be explained, perhaps, by rising unemployment observed among young people in Brazil. During the 90s, with the stabilization of inflation and fall in the level of economic activity, there was an increase in the unemployment rate, especially among younger workers. It has also contributed to this situation privatization and technological innovations and trade liberalization, resulting in increased informality and flexibility of labor relations. The presence of motorcyclists, popularly known as "couriers", and the trend growth of the services seem irreversible in the short and medium term, which may contribute to the increase of traffic accidents in the country. ${ }^{13,14}$

The results of this study regarding the predominance of young adults (mean age 30.7 years) of masculine gender (85\%) involved in motorcycle accidents, corroborate the results of Monk et al., ${ }^{15}$ who showed, in an epidemiological study held in the city of Alberta, Canada, that accidents involving individuals aged between 20 and 29 years represent the highest rate of mortality; these authors also observed that the registration of new motorcycles increased approximately $40 \%$ over the last 10 years, and relates closely to young and productive individuals. The vulnerability of motorcycle riders is evident, there is no protection similar to those of the occupants of four-wheeled vehicles. In a collision, the motorcyclist absorbs in his body surface all the energy generated at impact. Among various physical injuries that an individual can get after the accident, there are spinal cord injury, traumatic brain injury, and orthopedic injuries in lower and upper limbs. ${ }^{5}$

Individuals victim of motorcycle accidents, with injuries and acquired sequelae such as fractures, dislocations, severe wounds, amputation of limbs and plegia may have impairments in mobility, in occupational and everyday life, social relationships, physical and mental health.

In the present study we observed a higher involvement of the lower limbs (75.5\% of injuries), and this data may suggest a requirement for better protection of the region from the driver, and even of the vehicle. Another important preventive measure would be to implement traffic lanes exclusively for motorcyclists, as well as, rigorous inspection of personal protection instruments of the driver and the obedience of traffic rules. This would possibly decrease the risk of accidents.

According to Veronese et al, ${ }^{13}$ the risk of accidents is a complex phenomenon, which involves a network of interconnected possible damage to the individual: physical, emotional, legal, financial, moral and social. Since injured individuals are in a productive age, costs are high for both treatment and the loss of productivity. In the present study, for example, $95.4 \%$ of patients underwent surgical treatment and, consequently, underwent hospitalization, generating an estimated cost of $\mathrm{R} \$ 1,101,028.71$. However, the values shown are far from real, since they were estimated according to payment of the Unified Health System (SUS) to public hospitals, the costs were based on very low fees and value of subsidized implants, because it is a teaching hospital, the values of the implants are far below the market. The figures are alarming, and it is a public health situation, since we are facing the risk of death or serious sequelae, often permanently disabling. This issue is not a recent topic of discussion in the country, especially in large cities such as São Paulo, where increased traffic is imminent, including the increased use of motorcycles. Given this scenario, it is believed that the reduction of traffic accidents involving motorcyclists really depends on the adoption of effective preventive measures associated with the awareness of drivers and of the population.

\section{CONCLUSION}

According to the mapping of the profile of individuals involved in motorcycle accidents hospitalized in the Orthopaedics and Traumatology Ward of Hospital São Paulo, it was possible to identify some characteristics that may be useful for planning preventive strategies such as the development of protection mechanisms for lower limbs, stimulating the enforcement regarding the compliance of traffic laws by drivers, in an attempt to reduce the numbers of motorcycle accidents and redirect public investments in health. 


\section{REFERENCES}

1. Sinha AK, Boot DA, Gorman DF, Teanby DN. Severe motorcycle injury in Mersey region and North Wales. Injury. 1995;2 (8):543-5.

2. Wells S, Mullin B, Norton R, Langley J, Connor J, Lay-Yee R, Jackson R. Mo torcycle rider conspicuity and crash related injury: case-control study. BMJ. 2004;328(7444):857.

3. Alicioglu B, Yalniz E, Eskin D, Yilmaz B. Injuries associated with motorcycle accidents. Acta Orthop Traumatol Turc. 2008;42(2):106-111.

4. Silva DW, Andrade SM, Soares DA, Soares DF, Mathias TA. [Work profile and traffic accidents among motorcycle couriers in two medium-sized cities in the State of Paraná, Brazil]. Cad Saude Publica. 2008;24(11):2643-52.

5. Koizumi MS. Padrão das lesões nas vítimas de acidentes de motocicleta. Rev Saude Publica. 1992;26(5):306-15.

6. Santos AMR, Moura MEB, Nunes BMVT, Leal CFSL, Teles JBM. Profile of motorcycle accident victims treated at a public hospital emergency department. Cad Saude Publica. 2008;24 (8):1927-38.

7. Waiselfisz JJ. Mapa da violência 2011. Acidentes de trânsito. Brasília: Ministério da Justiça, Instituto Sangari; 2011.

8. Jeffers RF, Boon Tan H, Nicolopoulos C, Kamath R, Giannoudis PV. Prevalence and patterns of foot injuries following motorcycle. J Orthop Trauma. 2004;18(2):87-91.
9. Orsay E, Holden JA, Williams J, Lumpikin JR. Motorcycle trauma in the state of Illinois: Analysis of the Illinois department of public health trauma registry. Ann Emerg Med. 1995;26(4):455-60.

10. Aare M, von Holst H. Injuries from motorcycle and moped crashes in Sweden from 1987 to 1999. Injury control and safety promotion. Inj Control Saf Promot. 2003;10(3):131-8.

11. Tham KY, Seow E, Lau G. Pattern of injuries in helmeted motorcyclists in Singapore. Emerg Med J. 2004;21(4):478-82.

12. Ankarath S, Giannoudis PV, Barlow I, Bellamy MC, Matthews SJ, Smith RM. Injury patterns associated with mortality following motorcycle crashes. Injury 2002;33(6):473-7.

13. Veronese AM, Oliveira DLLC. Traffi c accidents from the motorcycle couriers' perspective: feedback for health promotion. Cad Saude Publica. 2006;22(12):2717-21.

14. Liberatti CLB, Andrade SM, Soares DA. The new Brazilian traffic code and some characteristics of victims in southern Brazil. Inj Prev. 2001; $7(3): 190-3$

15. Monk JP, Buckley R, Dyer D. Motorcycle-related trauma in Alberta: a sad and expensive story. Can J Surg. 2009;52 (6):235-40. 